\title{
HEP quantification strategy based on modified CREAM
}

\author{
PAN Xing*, WANG Huixiong, LIN Ye, LIU Tun, and WANG Xiaoxia
}

School of Reliability and Systems Engineering, Beihang University, Beijing 100191, China

\begin{abstract}
The accurate quantification of human error probability (HEP) has long been a main pursuit for most human reliability analysis (HRA) methods. This paper proposes a strategy to analyze and predict human error on the basis of a further modification of the existing cognitive reliability and error analysis method (CREAM) in HRA. Through providing a broader definition for the key parameters used in the quantification method, this paper produces a relatively flexible strategy to determine the nominal HEP $\left(\mathrm{HEP}_{0}\right)$. Basing on this strategy, the actual quantification of HEP in CREAM is able to be applied to more verified conditions. To prove the validity of the method proposed, a case study of spacecraft launch is also introduced, in which the prediction derived from the method is proved consistent with real field data.
\end{abstract}

Keywords: human reliability, cognitive reliability and error analysis method (CREAM), context influence, nominal human error probability $\left(\mathrm{HEP}_{0}\right)$.

DOI: $10.21629 /$ JSEE.2019.04.18

\section{Introduction}

Among all the efforts put into human reliability analysis (HRA) study, the accurate analysis and quantification of human error have always been emphasized and are critical to reduce and prevent human error behavior in practice. Despite all the progresses that have been made in the brief history of HRA, the general method for quantification of human error probability (HEP) has remained the same, which relies highly on the modification of the nominal HEP $\left(\mathrm{HEP}_{0}\right)$ and confines the influential factors to the task context. As a result, the correct value of critical parameters in such quantification can directly help determine the accuracy of the quantification result. The authors believe that a good strategy for quantifying HEP in the HRA method can provide a broad scheme for the selection of critical parameters and can adjust the calculation according to specific tasks. This paper proposes a modified method of HEP prediction based on the cognitive reliability and error

\footnotetext{
Manuscript received January 12, 2018.

*Corresponding author.

This work was supported by the National Natural Science Foundation of China (71171008; 71571004).
}

analysis method (CREAM), which has been considered one of the most well-developed HRA methods.

Based on the contextual control model (COCOM), Hollnagel [1] firstly proposed CREAM for general application, emphasizing the identification and quantification of cognitive errors from the perspective of information processing. The COCOM approach is constructed on the basis of the assumption that the environment or context is a major factor that affects HEP. CREAM establishes links between the operator's cognitive grade and HEP and puts the probability of the occurrence of the operator's behaviour under different control modes [2]. In CREAM, to achieve the quantification result of HEP, the tasks that relate to human cognition should be identified at the very beginning. After that, the context of these tasks is determined by the nine common performance conditions (CPCs). Then, the relationship between the task context and the human performance, which has been characterized as four control modes, can be built.

Although it has been decades since CREAM was put forward, relative studies have been improving this method. For one thing, new approaches such as fuzzy mathematics and neural network have been incorporated into the traditional CREAM [3-5]; for another, the modified CREAM has been applied to broader engineering fields like aerospace, medicine and so on [6,7]. In the existing modified CREAM method, a basic nominal HEP $\left(\mathrm{HEP}_{0}\right)$, which corresponds to the balanced working context, is defined, and the estimated HEP is determined by quantifying the difference between the actual working context and the balanced working context $[8,9]$. As a matter of fact, CREAM has been applied in some context of HRA. However, the determination of $\mathrm{HEP}_{0}$ lacks preciseness, which is the reason why the result of CREAM lacks preciseness and cannot be applied extensively [10]. Our study reveals that there exist two significant flaws in the existing methodology of CREAM. One is that the definition of the nominal context is incomplete and $\mathrm{HEP}_{0}$ can only be reached through the fixed numerical relationship between $\mathrm{HEP}_{0}$ and CPCs. The other is that there still remains some pos- 
sibility to simplify the quantification process in the developed CREAM, so that the relationship between the working context and specific conditions will be decided with more preciseness. The fundamental solution of these flaws is to determine a more effective $\mathrm{HEP}_{0}$, to correct $\mathrm{HEP}_{0}$ according to the analysis of CPCs, and to obtain HEPs under different working contexts [11].

To improve the flaws of the existing CREAM above, the authors propose a modified CREAM for working context analysis and HEP prediction. Specifically, the authors provide a comprehensive definition of $\mathrm{HEP}_{0}$ and the corresponding working context. A series of methods are constructed for determining the balanced working context so that the quantified CREAM can be applied to more scenarios of HEP prediction. Also, the authors use the proposed method to analyze a simple case study in commanding the fuelling process of spacecraft launch. The real field data are introduced to compare with the results from the proposed method, and the advancement of our proposed method is verified both in quantity and quality.

This paper is organized with the following parts: in Section 2, the authors dissect the existing CREAM and analyses the flaws in the definition of nominal HEP; the proposed modified CREAM is introduced in Section 3, where the modifications to the existing methods are emphasized; Section 4 illustrates the process of the proposed method with a simple case study in spacecraft launch, and the validity and preciseness of the proposed method is verified with the real field data; conclusions are presented in Section 5 .

\section{Existing CREAM and its flaws}

In this section, the existing CREAM method is introduced and its shortcomings that determine the working context are analyzed.

The main idea of the existing CREAM can be presented as (1) [8], which shows the relationship between the nominal $\mathrm{HEP}_{0}$ under the balanced working context and the actual HEP under the specific working context. This function is usually used to provide quantification predictions of performance reliability in probabilistic safety assessment (PSA), as shown in

$$
\ln \frac{\mathrm{HEP}}{\mathrm{HEP}_{0}}=k_{1} \beta
$$

where $k_{1}$ is a constant value, and $\beta$ is a parameter that contains the information of the working context. To be more specific, $\beta$ is defined as the difference between the sum of reduced CPCs (the sum of CPCs that deteriorates the working context, denoted as $\Sigma$ Reduced) and improved CPCs (the sum of CPCs that improves the working context, denoted as $\Sigma$ Improved). In the nominal context, sReduced $=\Sigma$ Improved $=0, \beta=0$, and therefore
$\mathrm{HEP}=\mathrm{HEP}_{0}$. When the reduced CPCs and the improved $\mathrm{CPCs}$ are considered corresponding with the working context, $\beta$ will change and therefore influence the relationship between $\mathrm{HEP}_{0}$ and HEP.

In CREAM, the condition of the actual working context is aggregated into nine CPCs, and the CPCs can be intervened and manipulated to ensure appropriate working circumstance. Specifically speaking, the working condition can be presented as the point $P$ ( $\Sigma$ Improved, $\Sigma$ Reduced $)$ on a two-dimensional coordinate system, which determines the actual HEP among all the possibilities of the working condition. Correspondingly, the nominal point $P_{0}$ (nominal $\Sigma$ Improved, nominal $\Sigma$ Reduced), also denoted as $P_{0}(\gamma, \eta)$, presents the balanced working context, where the task is expected to be performed normally without any intervention or manipulation. Therefore, $P_{0}(\gamma, \eta)$ determines the value of $\mathrm{HEP}_{0}$. In the existing CREAM, $P_{0}$ is assigned $(0,0)$.

To coordinate with the definition of $\mathrm{HEP}_{0}$, a modification has been made, in which $\mathrm{HEP}_{0}$ is decided exponentially by the nominal context and the HEP varies under the context. It can be shown in

$$
\mathrm{HEP}=k_{2} \cdot \exp (\mu w)
$$

where $k_{2}$ and $\mu$ are constant parameters, and $\omega$ contains the information of how harsh or how safe the working context is. Mathematically,

$$
\omega=\frac{\sum \text { Improved }}{\max \left(\sum \text { Improved }\right)}-\frac{\sum \text { Reduced }}{\max \left(\sum \text { Reduced }\right)} \text {. }
$$

It is assumed that max $\Sigma$ Improved $=7$ and $\max \Sigma$ Reduced $=9$, accord with the definition of control modes and the assumptions in the existing CREAM [1].

The CPCs in CREAM are used to depict the influential factors in a working context. According to previous assumptions of CREAM, if the working context ( $\Sigma$ Improved, $\Sigma$ Reduced) has no influence on the reliability of the operator's performance at all (i.e., $\Sigma$ Improved $=$ $\Sigma$ Reduced $=0$ ), the context will be balanced, under which the HEP is denoted as $\mathrm{HEP}_{0}$. It is also stated in [8] that $\mathrm{HEP}_{0}$ can be obtained when $\Sigma$ Reduced equals $\Sigma$ Improved (or all of the CPCs are insignificant). Considering the fact that different CPCs have varying influences on human performances, a more flexible strategy has been put forward [9] and a sound $\mathrm{HEP}_{0}$ in this strategy is determined when all CPCs are insignificant.

However, the authors believe that there are two flaws in the definition of $\mathrm{HEP}_{0}$ and $P_{0}(\gamma, \eta)$, which have limited the scope of the existing CREAM.

Flaw 1 The total number of nominal improved CPCs can be larger than 1 . The working process is not only a degradation process of machines, but also a fatigue process of operators. In this process, the reliability of human 
performance tends to reduce. In practical engineering industries, especially in those of high risks, including aeronautics [12], aviation [13], and marine [3] industries, the working conditions are usually of higher quality than others. Some of their insignificant CPCs are not stable and will easily turn into reduced CPCs. Therefore, improvements are made to ensure an appropriate working context, thus a higher number of improved CPCs will be acquired. Even if all CPCs are insignificant, their working conditions may still not be balanced. The nominal $P_{0}(\gamma, \eta)$ shall be flexible and closely related to experience data of a specific event. For some high-risk industries, $P_{0}(0,0)$ cannot ensure a balanced working context. For example, the manned Mars mission is full of risks and uncertainties. Everything in this mission could and would be done in accordance with the state-of-the-art and best practices in the field [14,15].

Flaw 2 In the existing CREAM, $P_{0}(\gamma, \eta)$ is assigned $(0,0)$, which excludes the situation that some CPCs may have greater effects on human performance than others. In some cases, it makes sense that some improved or reduced CPCs can compensate each other. Take the landing process of the passenger spacecraft as an example. Unexpected wind disturbances or aerodynamic responses derived from ground effect can be a big obstacle for pilots' visual landing (reduced CPC-working conditions) [16], but advanced instrument landing system (ILS) greatly helps cover this deficiency, which finally ensures a normal working context for the landing process (improved CPC-adequacy of man machine interface (MMI) and operational support). Therefore, it is possible to see $\gamma>0$ and $\eta>0$ in a balanced working context.

On the ground of the above two facts, the authors propose a strategy based on the modified CREAM in the next section. In this proposed CREAM, a series of rules are set to decide $P_{0}(\gamma, \eta)$. When $P_{0}$ is assigned according to the actual working context, the authors can better understand and analyze the relationship between CPCs and $\mathrm{HEP}_{0}$, and thus provide a precise and quantified prediction of HEP.

\section{Modified quantitative CREAM}

In this section, the authors introduce the proposed modified CREAM, and the effects of the modifications are illustrated with a quantified comparison.

In order to overcome the shortcomings of the existing CREAM in interpreting the relationship between the task context and $\mathrm{HEP}_{0}$, some modifications are made to that CREAM. The authors first modify the determination of control modes. In the proposed CREAM, the distribution interval of $P$ ( $\Sigma$ Improved, $\Sigma$ Reduced) should be further divided into two parts: safe (S) area and risky (R) area. Fig. 1 illustrates the coordinate plane of the working context, where the relationship between the context division and the distribution of $P(\Sigma$ Improved, $\Sigma$ Reduced $)$ can be found and explained.

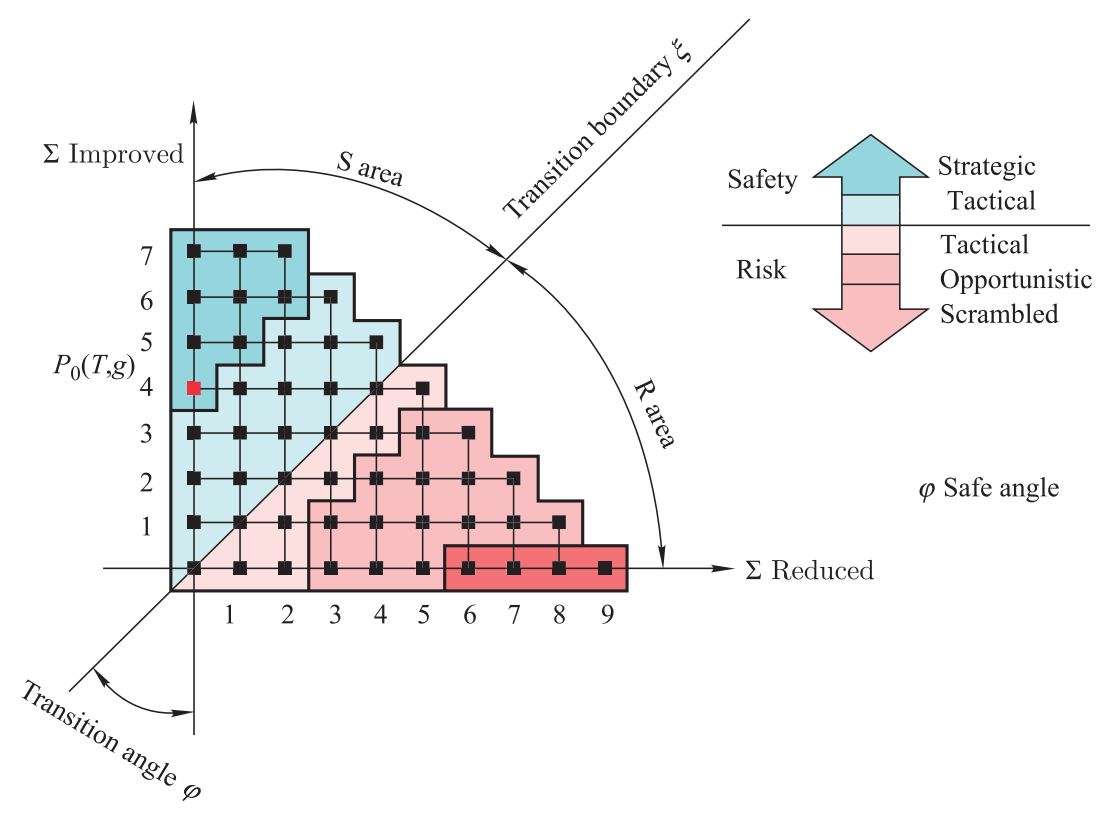

Fig. 1 Context division and control modes

Combined with the massive studies and the statement above, the authors make a brief description of the $\mathrm{S}$ area and the $\mathrm{R}$ area as follows: (i) the $\mathrm{S}$ area refers to the field where $\Sigma$ Improved is no smaller than $\Sigma$ Reduced. The R area represents the field where the number of $\Sigma$ Improved is smaller than that of 
sReduced.

(ii) The nominal $P_{0}(\gamma, \eta)$ is expected to locate in the $\mathrm{S}$ area, under which the balanced working context will lower the risk of human error.

(iii) When point $P(\Sigma$ Improved, $\Sigma$ Reduced) locates in the $\mathrm{S}$ area, the corresponding working condition will be in a relatively safe status. Otherwise, the working condition will be in a relatively risky status.

(iv) The CPCs will determine the position of the point $P$ in the context division. Under the balanced working context, $P=P_{0}$. When the working condition is controlled and modified, the CPCs will change, and thus the point

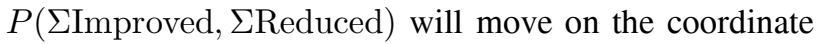
plane, resulting in a safe or risky working context.

In Fig. 1, the transition boundary $\xi$ divides the working context into the $\mathrm{S}$ area and the $\mathrm{R}$ area. The transition angle $\varphi$ is an included angle between the transition boundary $\xi$ and the vertical axis labeled as $\Sigma$ Improved. Basing on the descriptions of the $\mathrm{S}$ area and the $\mathrm{R}$ area mentioned above, it is obvious that the transition angle $\varphi=\pi / 4$.

Compared with previous quantified CREAM in $[8,9]$, the authors propose a much more flexible definition for the balanced context influence status $x$, as indicated in (4). $x$ here not only takes the contemporary working context of ( $\Sigma$ Improved, $\Sigma$ Reduced) into consideration, but the dynamic $P_{0}(\gamma, \eta)$ as well.

$$
x=\frac{\left(\sum \text { Improved }+\gamma\right)}{\max \left(\sum \text { Improved }\right)}-\frac{\left(\sum \text { Reduced }+\eta\right)}{\max \left(\sum \text { Reduced }\right)}
$$

where $\max \Sigma$ Improved $=7, \max \Sigma$ Reduced $=9$.

Referring to (1) and (2), the authors propose a more general equation to describe the exponential relationship between $x$ and HEP, as indicated in

$$
\mathrm{HEP}=k \cdot \boldsymbol{a}^{\lambda x}
$$

where $k, \boldsymbol{a}$, and $\lambda$ are model parameters, and $x$ contains information about the contemporary working context.

As described above, $\mathrm{HEP}_{0}$ reflects the basic HEP in which tasks are performed normally under a balanced situation. Fig. 1 illustrates the significance of selecting an appropriate nominal $P_{0}$. The relationship between each control mode and the corresponding HEP interval is shown in Table $1[1]$.

Table 1 Relationship between each control mode and HEP interval [1]

\begin{tabular}{cc}
\hline Control mode & HEP interval \\
\hline Strategic & $(0.00005,0.00100)$ \\
Tactical & $(0.00100,0.10000)$ \\
Opportunistic & $(0.01000,0.50000)$ \\
Scrambled & $(0.10000,1.00000)$ \\
\hline
\end{tabular}

According to the above-mentioned analysis and discussions, the authors then propose a more general and practical quantified method of CREAM.

Basing on Table 1 [1] and (5), we have

$$
\left\{\begin{array}{l}
\mathrm{HEP}_{0}=k \boldsymbol{a}^{\lambda\left(\frac{\gamma}{7}-\frac{\eta}{9}\right)}, \quad \mathrm{P}_{0}=(\gamma, \eta) \\
\mathrm{HEP}_{\min }=5 \times 10^{-4}, \quad \mathrm{P}_{\min }=(7,0) \\
\mathrm{HEP}_{\max }=1, \quad \mathrm{P}_{\max }=(0,9) \\
\left(\sum \mathrm{Improved}=\sum \text { Reduced }=0\right)
\end{array}\right.
$$

Equation set (6) can be further expressed as (7a) to (7c):

$$
\left\{\begin{array}{l}
\mathrm{HEP}_{0}=k \cdot \boldsymbol{a}^{\lambda\left(\frac{\gamma}{7}-\frac{\eta}{9}\right)} \\
k=\boldsymbol{a}^{\lambda\left(\frac{7-0}{7}-\frac{0-0}{9}\right)}=0.00005 \\
k=\boldsymbol{a}^{\lambda\left(\frac{0-0}{7}-\frac{9-0}{9}=1\right.}
\end{array}\right.
$$

According to $\frac{\text { Equation }(7 \mathrm{~b})}{\text { Equation }(7 \mathrm{c})}$, we have

$$
\begin{gathered}
\boldsymbol{a}^{\lambda}=7.07 \times 10^{-3} \\
k=7.07 \times 10^{-3} .
\end{gathered}
$$

Then, basing on (4) - (7), the authors have (10) and (11):

$$
\begin{gathered}
\mathrm{HEP}=\mathrm{HEP}_{0} \cdot\left(7.07 \times 10^{-3}\right)^{\left(\frac{\sum \text { Improved }}{7}-\frac{\sum \text { Reduced }}{9}\right)} \\
\mathrm{HEP}_{0}=\left(7.07 \times 10^{-3}\right)^{\left(\frac{\gamma}{7}-\frac{\eta}{9}+1\right)} .
\end{gathered}
$$

Finally, from (10) and (11), the eventual expression of HEP is

$$
\mathrm{HEP}=\left(7.07 \times 10^{-3}\right)^{\left(\frac{\sum \text { Improved }+\gamma}{7}-\frac{\sum \text { Reduced }+\eta}{9}+1\right)} .
$$

According to (11), it can be seen obviously that $\mathrm{HEP}_{0}$ is directly determined by the nominal point $P_{0}(\gamma, \eta)$, while HEP is determined by both $P_{0}$ and P. However, neither $\mathrm{HEP}_{0}$ nor HEP has any relationship with the parameter $a$, which means that the value of $a$ has no effect on the measurement of HEP. Therefore, the selection of the value of $a$ could be flexible in this modified CREAM quantification process.

Fig. 2 illustrates the distribution of HEP when $\Sigma$ Improved $+\gamma$ and $\Sigma$ Reduced $+\eta$ vary. By comparing (11) with (12), it can also be concluded that $\mathrm{HEP}_{0}$ is on the surface of HEP distribution. As shown in Fig. 2, when $\Sigma$ Improved $+\gamma=\Sigma$ Reduced $+\eta, \mathrm{HEP}_{0}$ meets the requirement for $\mathrm{HEP}_{0}$ in [8]. And its corresponding point $\left(P, \mathrm{HEP}_{0}\right)$ locates on a line (denoted by $-\times-\times-$ in Fig. 2$)$; when $\Sigma$ Improved $+\gamma=\Sigma$ Reduced $+\eta, \mathrm{HEP}_{0}$ meets the requirement for $\mathrm{HEP}_{0}$ (denoted by a round dot in Fig. 2) in [9]. In this paper, the point $\left(P_{0}, \mathrm{HEP}_{0}\right)$ is adjustable on the surface of the $\mathrm{S}$ area. Under this definition, both He's [8] and Sun's [9] definitions of $\mathrm{HEP}_{0}$ are included as special cases here. Furthermore, Fig. 2 also indicates that an appropriate $\mathrm{HEP}_{0}$ can also be determined. Take a nominal point $P_{0}(4,0)$ for example, the authors can easily know that $\mathrm{HEP}_{0}=4.17365 \times 10^{-3}$ (denoted by a square dot). 


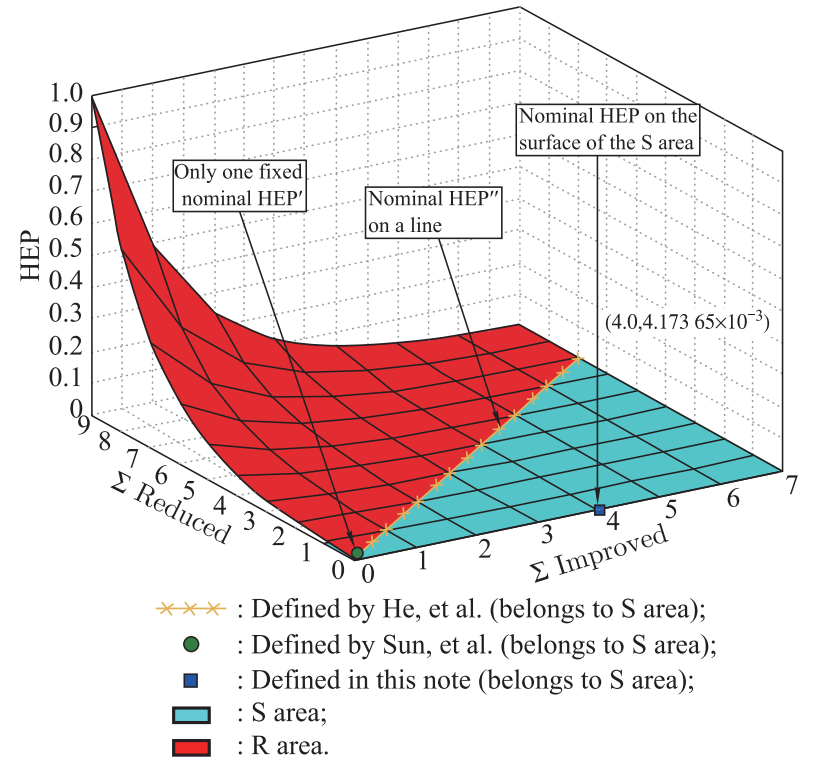

Fig. 2 Relationship between HEP and CPCs

To illustrate the differences between the proposed method and the existing one, the authors build the follow- ing equation to calculate the differences between the HEPs derived from the two methods. Use the case that $P_{0}(\gamma, \eta)$ is assigned $(4,0)$ as an example.

$\Delta_{\sum \text { Improved } \sum \text { Reduced }}=$
$\operatorname{HEP}_{\sum \text { Improved } \sum \text { Reduced }, \gamma, \eta}-\operatorname{HEP}_{\sum \text { Improved } \sum \text { Reduced }}^{\prime}$

where

$$
\begin{gathered}
\mathrm{HEP}^{\prime}=0.007071 \\
\exp \left[-4.9517\left(\frac{\Sigma \text { Improved }}{7}-\frac{\Sigma \text { Reduced }}{9}\right)\right]
\end{gathered}
$$

was firstly put forward by Sun et al. [9]; $\Delta$ represents the contemporary mathematical difference of HEP between the proposed method and Sun's.

The calculation result of (13) is listed in Table 2. By observing the results in Table 2, the authors learn that

$$
|\Delta|_{\max }=0.543 \cdot\left(\sum \text { Improved }=0, \sum \text { Reduced }=8\right)
$$

\begin{tabular}{|c|c|c|c|c|c|c|c|c|c|c|}
\hline \multirow{2}{*}{$\Sigma$ Improved } & \multicolumn{10}{|c|}{$\Sigma$ Reduced } \\
\hline & 0 & 1 & 2 & 3 & 4 & 5 & 6 & 7 & 8 & 9 \\
\hline 0 & -0.00665 & -0.01153 & -0.01999 & -0.03466 & -0.06009 & -0.10416 & -0.18057 & -0.31304 & -0.54268 & - \\
\hline 1 & -0.00328 & -0.00568 & -0.00986 & -0.01708 & -0.02962 & -0.05134 & -0.08902 & -0.15431 & - & - \\
\hline 2 & -0.00162 & -0.00280 & -0.00486 & -0.00842 & -0.01460 & -0.02531 & -0.04388 & - & - & - \\
\hline 3 & -0.00080 & -0.00138 & -0.00239 & -0.00415 & -0.00720 & -0.01248 & - & - & - & - \\
\hline 4 & -0.00039 & -0.00068 & -0.00118 & -0.00205 & -0.00355 & - & - & - & - & - \\
\hline 6 & -0.00010 & -0.00017 & -0.00029 & - & - & - & - & - & - & - \\
\hline 7 & -0.00005 & -0.00008 & - & - & - & - & - & - & - & - \\
\hline
\end{tabular}

Table 2 Mathematical difference between Sun's and the proposed HEP

Equation (16) indicates that the differences between these two quantified methods cannot be ignored. To avoid redundancy, the authors would not introduce traditional HRA methods, such as the technique for human error rate prediction (THERP) and human errer assessment and reduction technique (HEART), to validate (14).

\section{A simple case analysis}

In this section, the authors will present a simple case study to illustrate the approach of the proposed modified CREAM. Also, the improvements in the new approach are verified by comparing the results of the proposed method with that of the real field data of human reliability.

The targeted scenario the authors have chosen is the fueling process prior to the launch of spacecraft. Typically, this task demands a group of crew members to cooperate with each other and each one of them is labeled with a specific number, as indicated in Fig. 3.

In this cooperative task, there are ten crews being as- signed to different operations such as the power distribution, gas distribution, valve adjustment and so on. Another four crews are assigned to take care of fire protection, monitoring and information collection. Among all crews, one is supposed to hold the most crucial position as the commander, he or she is marked No. 0 at the top of Fig. 3.

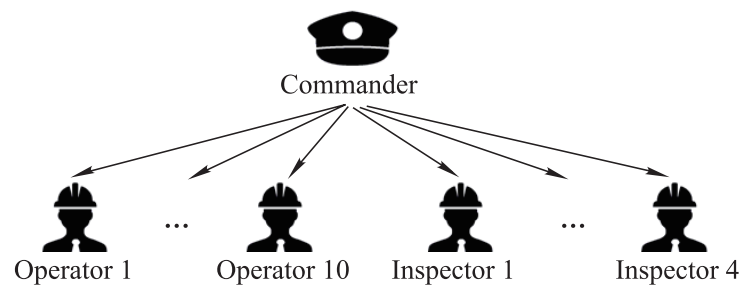

Fig. 3 Command relations in fueling process

The commander has to give correct and explicit orders to other crews at the exact point of the task time frame. The task of the commander involves plenty of cognition resources and can only be accomplished successively with 
high degree of concentration. In addition, as the fueling process is actually related to various influential factors during the launch of spacecraft, the specific task context of the commander is a set of extremely dynamic and complicated conditions.

In order to determine $P_{0}$ and $P$ for HEP prediction, a thorough evaluation of the working context is necessary. The authors refer to several experts' evaluations of the
CPCs from the Institute for Engineering Design and Research of the Chinese People's Liberation Army General Armaments Department, as shown in Table 3. In the last column, "I" means improved, "R" means reduced, and "NS" means not significant. As indicated in Table 3, the states of the nine CPCs for the commander's task have been determined, and therefore ( $\Sigma$ Improved, $\Sigma$ Reduced) is set as $(0,6)$.

Table 3 Evaluations on CPCs in task context

\begin{tabular}{|c|c|c|c|}
\hline $\mathrm{CPC}$ & Task context & Level & Effect \\
\hline $\begin{array}{l}\text { Adequacy of } \\
\text { organization }\end{array}$ & $\begin{array}{l}\text { As the commander holds the top priority in the crew group and takes full responsibility for all the } \\
\text { instructions in the fueling process, the relationship in the organization is not well-balanced. }\end{array}$ & Inefficient & $\mathrm{R}$ \\
\hline Working conditions & $\begin{array}{l}\text { The commander has to communicate with different operators from a far distance without knowing } \\
\text { the latest situation and the conditions in this environment can be considered as stressful and taxing. }\end{array}$ & Incompatible & $\mathrm{R}$ \\
\hline $\begin{array}{l}\text { Adequacy of MMI } \\
\text { and operational } \\
\text { support }\end{array}$ & $\begin{array}{l}\text { The keyboard buttons are too small and the commander has inadvertently touched the button in the } \\
\text { commanding task. }\end{array}$ & Inadequate & $\mathrm{R}$ \\
\hline $\begin{array}{c}\text { Availability of } \\
\text { procedures/plans }\end{array}$ & $\begin{array}{l}\text { The whole fueling process has been fully informed to all the crew members and the commander is } \\
\text { familiar with the processes. }\end{array}$ & Adequate & NS \\
\hline $\begin{array}{l}\text { Number of } \\
\text { simultaneous goals }\end{array}$ & $\begin{array}{l}\text { The commander has to monitor the performance of all the crew members while giving correct } \\
\text { instruction at the proper time. Also, if there is any emergency, the commander has to stop the } \\
\text { whole fueling process. }\end{array}$ & $\begin{array}{l}\text { More than } \\
\text { capacity }\end{array}$ & $\mathrm{R}$ \\
\hline Available time & $\begin{array}{l}\text { The commander is working in extremely high-pressure and in most cases. He or she may not have } \\
\text { any chance to correct the mistake in instruction as the time schedule is compact. }\end{array}$ & Inadequate & $\mathrm{R}$ \\
\hline $\begin{array}{c}\text { Time of day } \\
\text { (circadian rhythm) }\end{array}$ & $\begin{array}{l}\text { The exact time for the fueling task corresponds to the spacecraft launch and the command task can } \\
\text { be at any time of the day; thus, the commander may not be at his or her favorite circadian rhythm. }\end{array}$ & Unadjusted & $\mathrm{R}$ \\
\hline $\begin{array}{c}\text { Adequacy of } \\
\text { training and } \\
\text { experience }\end{array}$ & $\begin{array}{l}\text { The command needs to take comprehensive training to acquire the qualification for performing the } \\
\text { command task and most commanders have years of experiences. }\end{array}$ & Adequate & I \\
\hline $\begin{array}{l}\text { Crew collaboration } \\
\text { quality }\end{array}$ & $\begin{array}{l}\text { The crew members are capable of keeping communicating and cooperating with each in the fueling } \\
\text { task. }\end{array}$ & Adequate & NS \\
\hline
\end{tabular}

Another crucial improvement in the proposed method is the redefinition of $\mathrm{HEP}_{0}$. In this task context, as the commander is essential in the whole fueling process and has to face potential challenges such as interrupting the whole fueling process in emergency, some compensatory measures are taken to ensure an appropriate working context, which indicates that the level of CPCs in the balanced context is superior to the average.

According to the analyses of the experts, the commander's task is implemented with well-arranged operation procedures. Therefore, the level of nominal CPCavailability of procedures/plans is suggested to be appropriate instead of acceptable. Correspondingly, the nominal $P_{0}$ should be defined as $(1,0)$ instead of $(0,0)$. Therefore, it can be clearly seen from Fig. 1 that the defined $P_{0}$ locates in the $\mathrm{S}$ area, meeting with the normal situation which the authors have discussed in Section 2.2. Substituting $P_{0}=(1,0)$ into (13), we have

$$
\mathrm{HEP}_{0}=0.00348 \text {. }
$$

With this evaluation result substituted into (12), the authors obtain the following prediction of HEP:

$$
\mathrm{HEP}=\operatorname{HEP}_{0}\left(7.07 \times 10^{-3}\right)^{\left(\frac{\sum \text { Improved }}{7}-\frac{\sum \text { Reduced }}{9}\right)}=
$$$$
0.094609 \text {. }
$$

However, if $P(0,6)$ is assigned to the existing method proposed in [9], the prediction of HEP will be

$$
\mathrm{HEP}^{\prime}=0.007071 \cdot \mathrm{e}^{-4.9517\left(\frac{\sum \text { Improved }}{7}-\frac{\sum \text { Reduced }}{9}\right)}=
$$$$
0.191904 \text {. }
$$

Compared with the fixed $\mathrm{HEP}_{0}^{\prime} \equiv 7.07 \times 10^{-3}$ in previous studies [8,9], the new $\mathrm{HEP}_{0}$ for this task is significantly lower, which reflects a higher level of safety demands for human operation in spacecraft launch. From the fact that $\mathrm{HEP}_{0}<\mathrm{HEP}_{0}^{\prime}$, it can be concluded that the new definition for $P_{0}$ is stricter than the original one for $P_{0}^{\prime}$. Intuitively, when selecting the nominal point $P_{0}$ to present the balanced task context for such a high safety area, priority should be given to $P_{0}$, rather than $P_{0}^{\prime}$.

Apart from exhibiting the above results, real field data are also used to assess the validity of the results derived from the proposed method. The authors refer to the accident reports of human operations in Chinese spacecraft launch and have the following findings. 
In the eight satellite launch missions and one manned space mission in 2008, 2010 and 2011, there are 12 noted errors in the fueling processes caused by mis-instructions of the commander. As the command task of the fueling processes can be subdivided into 14 subtasks, there are $126(9 \times 14)$ chances in total for the commander to make mistakes. As a result, the authors can calculate that the error frequency of the command task is 0.095235 , which is significantly closer to the result derived from the proposed method.

In this case, it can be concluded that the results of the proposed method and the field data are close, and thus the proposed method can predict HEP and help build more efficient and dependable prevention measures in the actual human operations. Besides, the result produced by the new approach is closer to the actual HEP than the existing CREAM, which verifies the improvements in accuracy of this research.

\section{Conclusions}

Compared with previous quantification methods in CREAM, the authors provide a thorough illustration of critical parameters' modifications involved in the quantification. On the one hand, to broaden the limited assumptions of HEP with external influential conditions quantificationally, the authors adopt a more flexible method to select a constant value $a$. Basing on a broader value range of this basic parameter, the cognitive model in HRA can be adjusted easily in practical use, so that a more precise prediction of HEP can be obtained. On the other hand, the authors propose a comprehensive definition of $\mathrm{HEP}_{0}$ to distinguish the special requirements for context status in high-risk industries, which is actually a necessity for an adjustable $\mathrm{HEP}_{0}$ to correspond with different kinds of applied fields.

The authors make a complete presentation of the procedures in the proposed HEP quantification strategy of the modified CREAM. By applying this proposed strategy to command tasks in the fueling process of spacecraft launch, the consistency between the proposed method and previous efforts as well as the validity of this method in human error prediction are verified. More importantly, the final calculation result of this modified CREAM shows a significant improvement in the existing quantification methods.

\section{References}

[1] HOLLNAGEL E. Cognitive reliability and error analysis method (CREAM). Amsterdam: Elsevier, 1998.

[2] PAN X, LIN Y, HE C. A review of cognitive models in human reliability analysis. Quality and Reliability Engineering Inter- national, 2016, 33(7): 1299-1316.

[3] YANG Z, BONSALL S, WALL A, et al. A modified CREAM to human reliability quantification in marine engineering. Ocean Engineering, 2013, 58: 293-303.

[4] UNG S T. A weighted CREAM model for maritime human reliability analysis. Safety Science, 2015, 72: 144-152.

[5] MU L, XIAO B, YUAN Z, et al. The research of CREAM prediction analysis method based on BP neural network under dynamic context. Proc. of the 1st International Conference on Reliability Systems Engineering, 2015: 1-6.

[6] CALHOUN J, SAVOIE C, RANDOLPH-GIPS M, et al. Human reliability analysis in spaceflight applications, Part 2: modified CREAM for spaceflight. Quality and Reliability Engineering International, 2014, 30(1): 3- 12.

[7] DEETER J L. Human reliability analysis in healthcare: application of the cognitive reliability and error analysis method (CREAM) in a hospital setting. https://search.proquest.com/docview/1024285130.

[8] HE X, WANG Y, SHEN Z, et al. A simplified CREAM prospective quantification process and its application. Reliability Engineering \& System Safety, 2008, 93(2): 298-306.

[9] SUN Z, LI Z, GONG E, et al. Estimating human error probability using a modified CREAM. Reliability Engineering \& System Safety, 2012, 100: 28-32.

[10] LAUMANN K. Criteria for qualitative methods in human reliability analysis. Reliability Engineering \& System Safety, 2018, DOI: 10.1016/j.ress.2018.07.001.

[11] PAN X, WU Z. Performance shaping factors in the human error probability modification of human reliability analysis. International Journal of Occupational Safety and Ergonomics, 2018: $1-13$.

[12] CHANDLER F, CHANG Y, MOSLEH A, et al. Human reliability analysis methods: selection guidance for NASA. Washington, DC: NASA Office of Safety and Mission Assurance, 2006.

[13] FOYLE D C, HOOEY B L. Using human performance modeling in aviation. Boca Raton: CRC Press, 2007.

[14] SALOTTI J M, SUHIR E. Manned missions to Mars: minimizing risks of failure. Acta Astronautica, 2014, 93: 148 - 161.

[15] ILYIN E A, KHOLIN S F, GUSHIN V I, et al. Human factor in manned Mars mission. Advances in Space Research, 1992, 12(1): $271-279$.

[16] AMBATI P R, PADHI R. Robust auto-landing of fixed-wing UAVs using neuro-adaptive design. Control Engineering Practice, 2017, 60: $218-232$.

\section{Biographies}

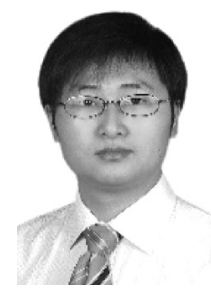

PAN Xing was born in 1979. He received his B.S. degree in mechanical engineering, and Ph.D. degree in systems engineering from Beihang University, Beijing, China, in 2000 and 2005 respectively. From 2005 to 2009, he was an assistant professor with the School of Reliability and Systems Engineering, Beihang University, Beijing, China. Since 2009, he has been an associate professor. From 2012 to 2013, he was a visiting scholar at the Department of Systems and Industrial Engineering, University of Arizona, Tucson, USA. He is currently an associate professor with the School of Reliability and Systems Engineering of Beihang University. His research interests include reliability engineering, systems engineering, and system risk analysis. E-mail: panxing@buaa.edu.cn 


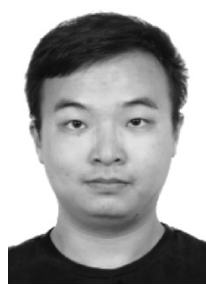

WANG Huixiong was born in 1995. He received his B.S. degree in safety science from School of Reliability and Systems Engineering, Beihang University, in 2018 . He is currently a master student in the same school. His interests of research include systems engineering and complex network.

E-mail: wanghuixiong@buaa.edu.cn

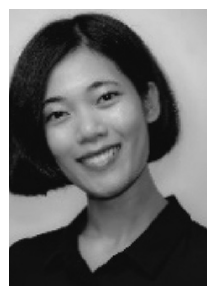

LIN Ye was born in 1993. She received her B.S. degree in aerospace engineering, and M.S. degree in systems engineering from Beihang University, Beijing, China, in 2015 and 2017 respectively. Currently, She is a Ph.D. candidate in systems engineering from Boston University, MA, USA. Her research interests include reliability engineering and systems engineering.

E-mail: yelin@bu.edu

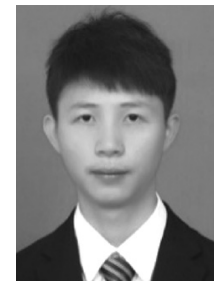

LIU Tun was born in 1996. He received his B.S. degree in mechanical science and engineering from Jilin University, Jilin, China, in 2017. He is currently pursuing his M.S. degree in reliability and systems engineering with the School of Reliability and Systems Engineering, Beihang University, Beijing, China. His research interests include human reliability analysis, reliability engineering, and system risk analysis.

E-mail: liu_tun@163.com

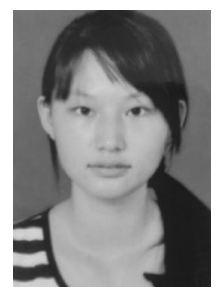

WANG Xiaoxia was born in 1994. She received her B.S. degree in safety engineering from China University of Mining and Technology, Jiangsu, China. She is currently pursuing her M.S. degree in reliability and systems engineering with the School of Reliability and Systems Engineering, Beihang University. Her research focus is human reliability, including quantification of human reliability for individuals and crews.

E-mail: langshixuan@foxmail.com 